\title{
Counteracting venous stasis during acute lower leg immobilization
}

\author{
F. Poelkens, ${ }^{1,2 *}$ D. H. J. Thijssen, ${ }^{1,2}{ }^{2}$ B. Kersten, ${ }^{1,2}$ H. Scheurwater, ${ }^{3}$ E. W. van Laarhoven ${ }^{4}$ \\ and M. T. E. Hopman ${ }^{1,2}$ \\ I Fysiorun, Research Centre, Radboud University Nijmegen Medical Centre, The Netherlands \\ 2 Department of Physiology, Radboud University Nijmegen Medical Centre, The Netherlands \\ 3 Department of Orthopaedic Cast Technology, TweeSteden Hospital, Tilburg/Waalwijk, The Netherlands \\ 4 Department of Orthopaedics, TweeSteden Hospital, Tilburg/Waalwijk, The Netherlands
}

Received 12 July 2005, revision requested 16 August 2005,

final revision received

3 October 2005, accepted 12 October 2005

Correspondence: M. T. E.

Hopman, Department of Physiology, RUN MC, PO Box 9101, 6500 HB Nijmegen,

The Netherlands.

E-mail: m.hopman@fysiol.umcn.nl *Both authors have contributed equally to this manuscript.

\begin{abstract}
Aim: During lower limb immobilization, patients are at risk to develop deep venous thrombosis. Recently, a water-pad was developed that should counteract venous stasis. The water-pad, located under the plaster, mobilizes water from the foot to the calf during weight bearing and, thereby, imitates muscle pump function. The purpose of this study was to assess the effect of the water-pad on venous pump function in healthy individuals.

Methods: In 21 healthy subjects (10 men and11 women) both legs were plastered. Venous pump function was assessed by plethysmography measuring lower leg venous ejection fraction and volume. Subjects were tilted from the supine position to upright standing to determine total venous volume. Hereafter, stepping was performed to measure venous ejection fraction and volume under different filling conditions of the water-pad $(0,50,100$, 150, 200, 250 and $300 \mathrm{~mL}$ ). Different sizes of water-pads (small, medium and large) were applied to each plastered leg in order to test the effectiveness and to relate optimum size to anthropometrical data.

Results: The venous ejection fraction increased significantly from $30 \pm 17 \%$ to a maximum of $42 \pm 19 \%$ during stepping with increasing filling condition (RM ANOva; $P=0.009$ ). Ejection volume also enhanced significantly during stepping with increasing filling condition from $1.3 \pm 0.7$ to $1.9 \pm 0.9 \mathrm{~mL}(100 \mathrm{~mL})^{-1}$ (RM Anova; $\left.P=0.006\right)$. The optimal filling condition of the water-pad depended on the water-pad size, while body height was the best predictive value for the water-pad size (Pearson's $R=$ $0.72, P<0.001)$.

Conclusion: The filled water-pad markedly increased the venous ejection fraction and volume of the lower leg during stepping, hereby counteracting stasis of venous blood in the immobilized lower leg. Therefore, the water-pad seems to be a promising tool to prevent deep venous thrombosis during periods of lower leg immobilization.

Keywords deep venous thrombosis, fracture, muscle pump activity, strain gauge plethysmography, venous ejection fraction, venous ejection volume.
\end{abstract}


Leg fractures, which are a common medical problem with an increasing incidence over the last few years (Leppilahti \& Orava 1998), are most commonly treated with prolonged immobilization. During this prolonged period of lower leg immobilization, deep venous thrombosis (DVT) or venous thrombo-embolism are frequently reported complications (Geerts et al. 1994, Jorgensen et al. 2002, Lassen et al. 2002, Anderson \& Spencer 2003), which eventually can result in a lifethreatening pulmonary embolism (Micheli 1975, Geerts et al. 1994, Anderson \& Spencer 2003). The risk of developing DVT during conventional lower limb immobilization varies between $4.5 \%$ (Zagrodnick \& Kaufner 1990) and 71.4\% (Hjelmstedt \& Bergvall 1968), depending on the indication for immobilization and the method of diagnosing DVT, indicating the importance of prevention of DVT during lower leg immobilization.

The most common preventive countermeasure of DVT is to inhibit the coagulation pathway by administrating low-molecular-weight-heparin (Kujath et al. 1993, Spannagel \& Kujath 1993, Handoll et al. 2002). Previous studies investigating the effect of low-molecular-weight-heparin indicated a tendency (Jorgensen et al. 2002) or significant reduction (Kock et al. 1995, Lassen et al. 2002) in the incidence of DVT after leg injury requiring immobilization. However, disadvantages of this method are heparin-induced thrombocytopenia (Warkentin \& Kelton 1991, Warkentin et al. 1995), bleeding complications (Muntz et al. 2004), and patients' compliance to the therapy. In addition, stasis of blood because of lack of the pumping action of the calf muscle in immobilized lower legs is undoubtedly the major factor contributing to the development of DVT (Mammen 1992). Several mechanical methods were developed to enhance venous blood flow in the immobilized leg by imitating the muscle pump function. For example, a pneumatic compression pump (Bulitta et al. 1996) placed on the plantar venous plexus or electrical stimulation of the foot (Kaplan et al. 2002) or calf (Klecker \& Theiss 1994) demonstrated an increase in venous blood flow of the lower limb. Both methods counteract venous stasis and, therefore, potentially reduce the risk of DVT. However, shortcomings of both methods are patients' skills to connect the apparatus, applicability in plaster cast, and patients' time and compliance.

To overcome shortcomings of previous methods, a new method to counteract venous stasis during lower leg immobilization has been developed; a water-pad (XLerator; Somas BV, St Anthonis, the Netherlands). This water-pad, which is placed between the skin and the plaster cast or walker, connects the sole of the foot and the dorsal side of the calf and is hypothesized to imitate the skeletal muscle pump during locomotion. It is suggested that during the stance phase the water-pad mobilizes fluid, which was collected at the sole of the foot, to the calf. Consequently, pressure increases around the calf and irradiates proximally. When pressure drops during the swing phase, water is assumed to return to the initial position under the foot. However, there is no scientific data present regarding this mechanism. Therefore, the aim of this study is to assess the effect of the water-pad on venous ejection fraction (EF) and volume of the lower leg in healthy subjects during acute lower leg immobilization and, in addition, to determine the optimal size and filling condition of the water-pad. We hypothesize that the water-pad will enhance leg venous outflow.

\section{Methods}

\section{Subjects}

Twenty-one healthy subjects (11 women and 10 men) with a mean age of $35.1 \pm 13.4$ years (mean $\pm S D$ ) participated in this study after providing written informed consent. Subjects were normotensive, nonmedicated, and were free of significant medical problems as assessed by medical history. Subjects with a history of DVT or other vascular problems were excluded from participation. Subject characteristics are shown in Table 1 . The study was approved by the Faculty Ethics Committee.

\section{Water-pad}

The plaster cast used in this study consists of four different layers with the recently developed lower leg water-pad (XLerator; Somas BV) added to it. The first layer is a tricot stocking (Tricofix, BSN Medical, Hamburg, Germany), followed by a layer of Synthetic Under Cast Padding (Cellona; Lohmann-Rauscher). This second layer is finished with Adhesive Edge Padding Cast on the heel and tibia side of the lower limb (Cellona; Lohmann-Rauscher). The third layer consists of a Pre-tape (Delta-Cast, BSN Medical, Hamburg, Germany) and the end layer consists of a Primacast $\left(3 \mathrm{M}^{\mathrm{TM}}\right.$ Health Care, Salt Lake City, UT,

Table I Subject characteristics $(n=21)$

\begin{tabular}{lc}
\hline Variables & Mean $\pm \mathrm{SD}$ \\
\hline Age, years & $35.1 \pm 13.4$ \\
Weight, kg & $71.9 \pm 11.2$ \\
Height, cm & $175 \pm 9$ \\
Systolic blood pressure, $\mathrm{mmHg}$ & $130 \pm 11$ \\
Diastolic blood pressure, $\mathrm{mmHg}$ & $77 \pm 8$ \\
Sport time, h week & -1 \\
\hline
\end{tabular}


Figure I Process of casting a lower leg with the water-pad: (a) water-pad; (b) third layer; (c) fourth layer; and (d) air valve, used for filling of the water-pad during experiments.

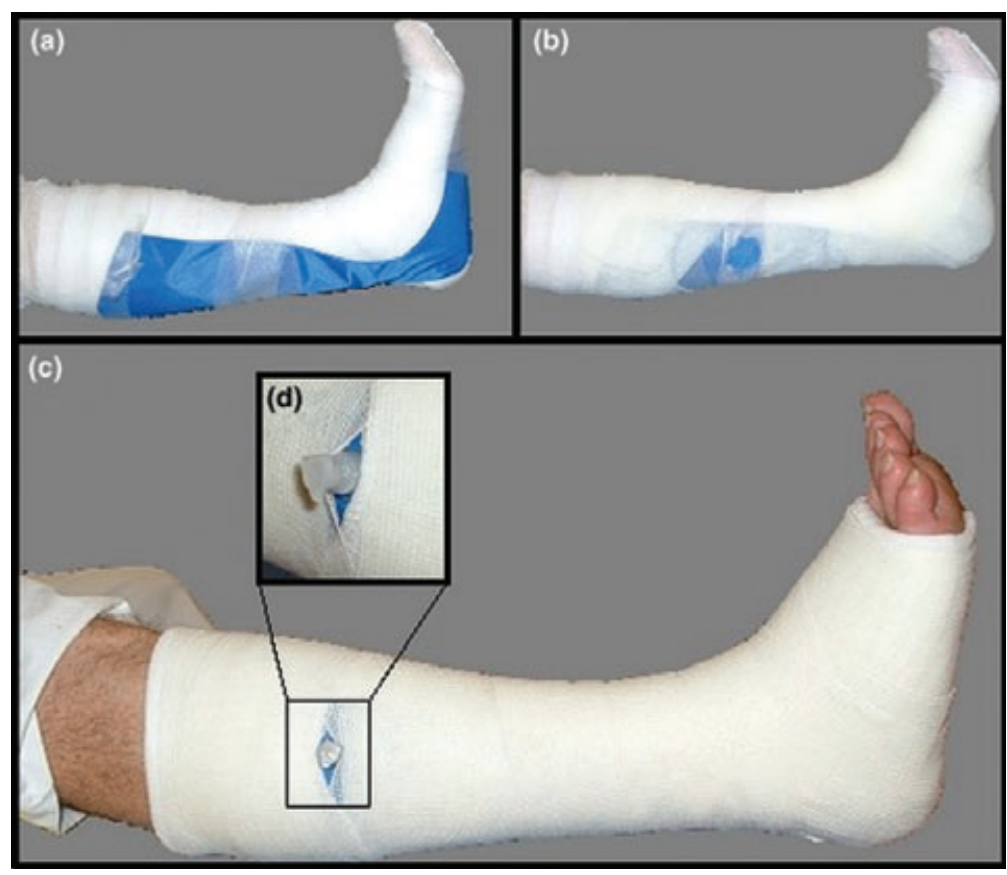

USA) and Circular Polycast $\left(3 \mathrm{M}^{\mathrm{TM}}\right.$ Scotchcast ${ }^{\mathrm{TM}}$ Health Care). Between the second and third layer the empty water-pad is placed under the foot and over the dorsal side of the calf. The water-pad is made of nylon with a coating of polyurethane. The water-pad can be filled with water via an air valve, specially developed for research purposes, placed at the dorsal side of the calf (Fig. 1).

\section{Protocol}

Subjects abstained from any food or beverages for at least $2 \mathrm{~h}$ before examination, and were not allowed to perform heavy exercise $24 \mathrm{~h}$ before testing to avoid the immediate effects of exercise. The measurements were performed in a quiet room, with a constant temperature between $22^{\circ}$ and $23^{\circ} \mathrm{C}$.

Previous to the assessment of venous volume (VV) changes, subjects were at least $25 \mathrm{~min}$ in the supine position for bilateral application of the lower leg cast immobilization. To determine the best size of the waterpad, two different sized water-pads were applied to each leg. For all subjects, on the right leg an empty, medium-sized water-pad was used. On the left leg, based on subjective judgement related to body size, an empty, small or large-sized water-pad was used.

Subjects lay on a tilt-table with their feet against a foot support. Mercury strain gauges venous plethysmography was used to measure lower leg volume changes, representing venous filling or emptying of the lower leg (van Beekvelt et al. 2000). During a resting period of at least $5 \mathrm{~min}$ in the supine position, strain gauges were positioned. The lower leg was slightly elevated to empty the calf venous system and obtain the estimated minimum calf volume. After a virtually stable baseline signal was achieved, neglecting the small and slow volume increase by fluid extravasation, testing procedures started. Subjects were tilted to the upright position. After a new virtually stable signal was achieved in the upright position, subjects had to perform 20 steps in $20 \mathrm{~s}$ to assess the effect of the water-pad and related water displacement on the venous $\mathrm{EF}$ (Stewart et al. 2004) and ejection volume (EV) (Nicolaides 2000) (Fig. 2). To correct for variation, this stepping-procedure was repeated three times with a resting period in between to assure a new stable signal in the upright position. Subsequently, subjects were tilted back to the supine position. To assess, the optimal filling of the water-pad, the whole testing procedure was repeated with increasing amounts of water in the water-pad with, respectively, 0, 50, 100, 150, 200, 250 and $300 \mathrm{~mL}$. Water-pads were filled in the supine position.

Based on the individual's largest increase in lower leg venous $\mathrm{EV}$, the most effective water-pad was chosen and was used for further analysis. Moreover, this most effective water-pad size was related to subjects' characteristics, such as weight, body height, lower leg length, shoe size, lower leg volume and ankle, calf and knee circumference. These anthropometrical variables were measured before testing.

\section{Measurements}

Venous plethysmography. The method to measure venous $\mathrm{EF}$ and $\mathrm{EV}$ was adapted from previous studies 


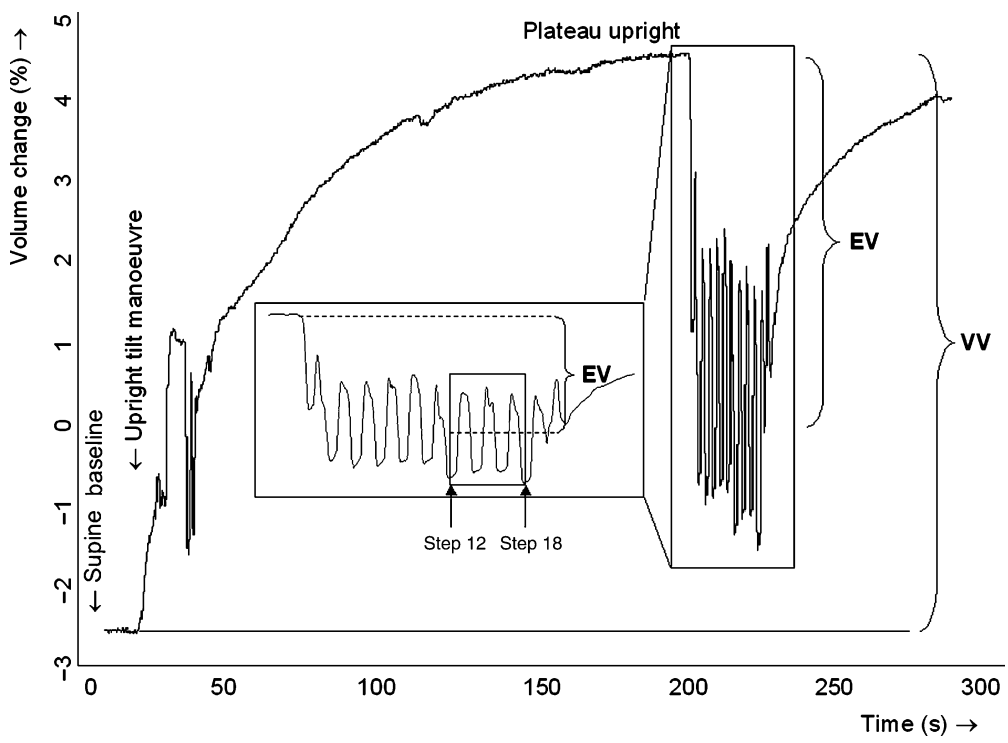

Figure 2 Typical plethysmographic recording measuring lower limb volume changes during a sequence of lying supine, upright tilt, and 20 steps. Ejection volume (EV) was calculated from the stable plateau condition in the upright position minus the average volume change between step 12 and 18 . The maximal venous volume $(\mathrm{VV})$ was defined as the maximal volume increase in the limb from the supine position to the stable plateau condition in the upright position. Venous ejection fraction $(\mathrm{EF})$ was defined as $[\mathrm{EV}$ (plateau upright - plateau steps)/VV (plateau upright - plateau supine) $] \times 100$.

(Nicolaides 2000, Stewart et al. 2004) and was measured by an automatic venous occlusion plethysmography (Hokanson, Bellevue, WA, USA) according to the principle described by Halliwill et al. (2002). The mercury-in silastic strain gauges (Hokanson) were placed $5 \mathrm{~cm}$ distal of the patella, just above the upper edge of the cast. Figure 2 represents a plethysmographic recording of the volume changes from supine to upright position and after 20 steps. From this recording, the venous EF can be calculated by dividing the venous EV by the VV; $(\mathrm{EV} / \mathrm{VV}) \times 100$. The VV $\left[\mathrm{mL}(100 \mathrm{~mL})^{-1}\right.$ tissue] is defined as the maximal volume increase in the limb from the supine position to the upright position. In addition, the venous EV [mL $(100 \mathrm{~mL})^{-1}$ tissue] was calculated from the plateau phase in the upright position minus the average volume change between step 12 and 18 . In a pilot study $(n=13$, data not shown), the coefficient of variation $(\mathrm{CV})$ of this method in our laboratory was found to be $15.8 \%$. The ratio of $\mathrm{EV} / \mathrm{VV}$, which is the EF (\% change from the VV), is used as a normalized index of skeletal muscle pump adequacy.

\section{Data analysis}

Plethysmographic data were digitalized with a sample frequency of $100 \mathrm{~Hz}$ (MIDAC, Instrumentation Department, Radboud University Nijmegen Medical Centre, the Netherlands) and analysed by a customized computer program (Matlab; Mathworks, Natick, MA, USA).

\section{Statistics}

Statistical analyses were performed using SPSS II.O computer software (SPSS, Chicago, IL, USA). Venous $\mathrm{EF}$ (\% from the lower leg VV) and EV [mL $\left.(100 \mathrm{~mL})^{-1}\right]$ for each individual filling condition was determined by averaging the results of the three repeated steppingtests. A two-way ANOva ('filling condition' as dependent variable, 'water-pad size' as independent variable) was used to assess the effect of the different water-pads on the venous EF and EV with increasing filling (SPSS Statistical Package). In addition, ANOvA post-hoc analysis was used to find the optimal filling for the small, medium and large sized water-pad. To relate anthropometrical data to the water-pad size, a Pearson's correlation coefficient was used. Data are presented as means \pm SD. The level of statistical significance was set at $\alpha=0.05$.

\section{Results}

\section{Venous ejection volume}

All subjects wore two sizes of water-pads, which were subdivided in the best- and less-effective water-pad. Venous EV of the lower leg without filling the waterpad, representing an ordinary cast, was $1.3 \pm$ $0.7 \mathrm{~mL}(100 \mathrm{~mL})^{-1}$. Using the best-effective waterpad, venous EV improved with increasing filling of the water-pad (ANOvA; $P=0.006$ ). There was no difference in $\mathrm{EV}$ between the three different water-pad sizes (ANova; $P=0.28$ ). The maximal venous $\mathrm{EV}$ was $1.9 \pm 0.8 \mathrm{~mL}(100 \mathrm{~mL})^{-1}$ with $150 \mathrm{~mL}$ filling, indicating a $41 \%$ increase compared with baseline values (ordinary cast) (Fig. 3). The less-effective water-pad did not demonstrate a significant improvement in venous EV (ANOVA; $P=0.43$ ).

Lower leg VV at the plateau phase in the upright position in subjects with the water-pad in the unfilled condition was $4.3 \pm 0.8 \mathrm{~mL}(100 \mathrm{~mL})^{-1}$. With increasing filling of the water-pad, lower leg VV did not change 

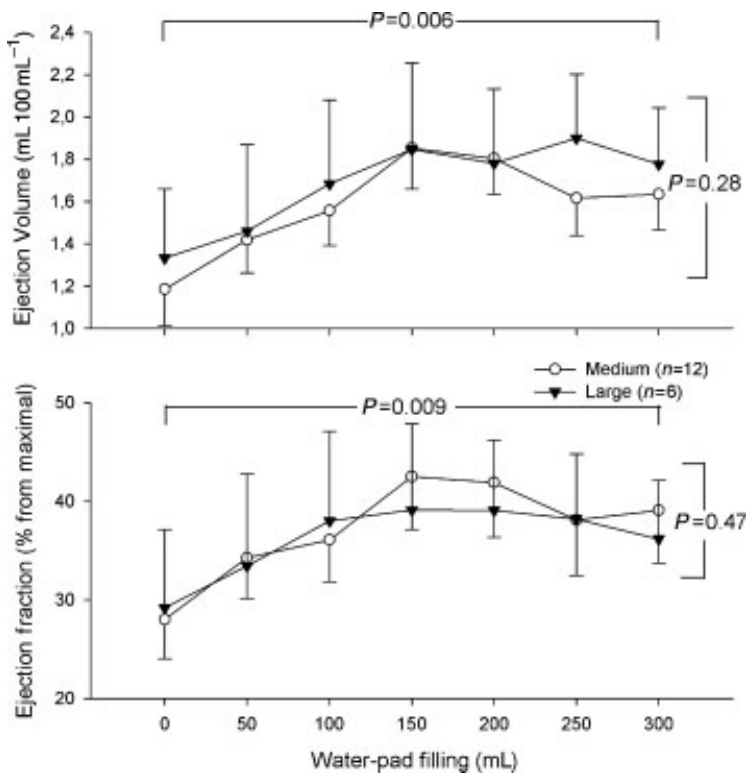

Figure 3 Venous ejection volume (EV; a) and fraction (EF; b) at different fillings of the water-pad $(n=21)$. Venous EV is indicated as the absolute change in venous volume $\left[\mathrm{mL}(100 \mathrm{~mL})^{-1}\right]$. Venous $\mathrm{EF}$ is presented as a per cent of the maximal venous volume. Only the best-effective size was represented. Each figure represents the medium and large sized water-pad (the small water-pad had an $n=3$, and is therefore left out). A two-way ANova (filling condition as within-subject factor, water-pad size as between-subject factor) was used to compare the changes in EF and EV with increasing filling condition and whether the change was different between the water-pad sizes (EF, ejection fraction; EV, ejection volume; error bars represent SEM).

(ANOVA; $P=0.16$ ) and did not differ among the three water-pads (ANOvA; $P=0.18$ ).

To find the optimal filling condition for the different sized water-pads, the water-pads were separately analysed. Post-hoc analysis for the medium sized water-pad $(n=12)$ indicated an optimal filling condition between 100 and $200 \mathrm{~mL}$ (150 mL: post-hoc significant from all conditions, except 100 and $200 \mathrm{~mL}$ ). Due to the relatively small group for the large water-pad $(n=6)$, the Post-hoc analysis was not accurate to indicate an optimal filling condition. The small water-pad $(n=3)$ was not included in the data presentation.

\section{Venous ejection fraction}

Venous EF of the lower leg without filling of the waterpad, representing an ordinary cast, was $30 \pm 17 \%$. With increasing filling of the water-pad, venous EF increased significantly up to $42 \pm 19 \%$ (ANOvA; $P=$ $0.009)$. There was no difference in venous EF between the different sized water-pads (ANOvA; $P=0.47$, Fig. 3). At $150 \mathrm{~mL}$ water-pad filling, the maximal

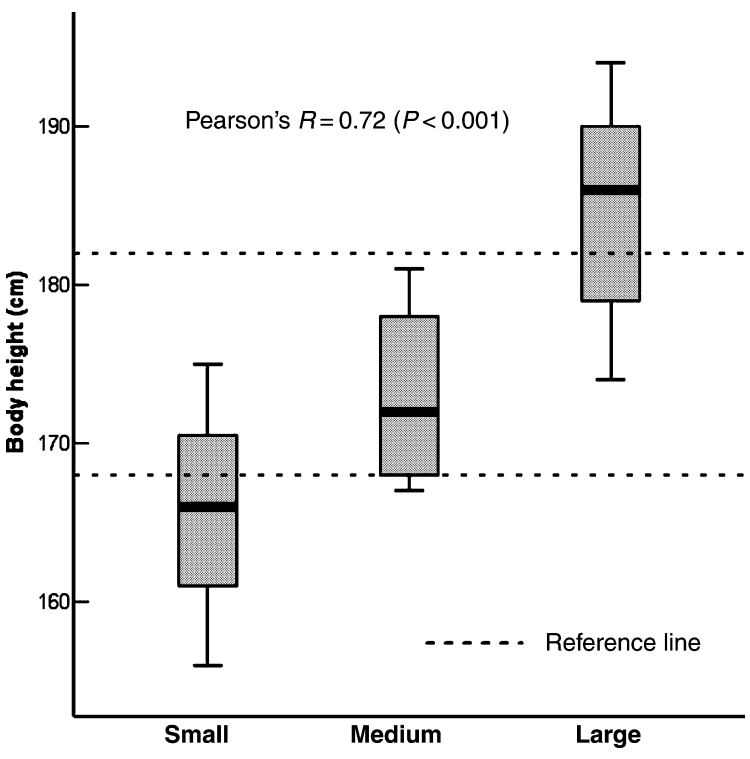

Figure 4 Correlation between body height and optimum water-pad size (Pearson's correlation coefficient). The black line represents the group's median. The borders of the grey box and error bars represent the interquartile ranges. The dotted lines represent the transition from small-medium and mediumlarge water-pad size.

venous EF was achieved. The less-effective size of the water-pad showed no change in venous EF (ANOvA; $P=0.25)$. Post-hoc analysis for the venous EF, used to find the optimal filling condition for the water-pad, showed results similar to the Post-hoc analysis for venous $\mathrm{EV}$.

\section{Anthropometrical data}

Body height showed the highest correlation with the optimum water-pad size (Pearson $R=0.72, P<0.001$, Fig. 4). Lower leg length and ankle circumference showed a significant correlation with the optimum water-pad size as well (Pearson $R=0.55$ and 0.50 respectively, $P<0.05)$. Calf and knee circumference, lower leg volume and shoe size did not show a significant correlation with the optimum water-pad size.

\section{Discussion}

The primary findings from the present study are the following: (1) the filled water-pad causes a significant increase in venous EV and EF of the lower leg; (2) a water-pad filling of $150 \mathrm{~mL}$ is recommended, as venous $\mathrm{EV}$ and fraction were significantly related to the degree of filling of the water-pad between 0 and $150 \mathrm{~mL}$; and (3) body height is a good predictive value for choosing the best water-pad size. The combination of plaster cast and water-pad successfully enhances lower leg venous outflow in healthy individuals. 
The suggested working mechanism of the water-pad, mimicking the muscle pump activity, is based on the assumption that muscle pump activity is impaired during lower leg immobilization, leading to venous stasis. Previous studies showed plaster-immobilization to result in vascular atrophy (Sugawara et al. 2004), decrease in muscle strength (Booth 1987, Kitahara et al. 2003), and altered muscle composition (Booth 1987), all indicative for attenuated muscle activity. In a pilot study, we tested lower leg muscle activity, expressed as electromyography (EMG) activity, of the medial gastrocnemius muscle with and without a below-the-knee cast $(n=4)$. We demonstrated a $34 \%$ reduction in EMG activity in a below-the-knee plaster cast (paired $t$-test; $P=0.001)$ during walking $\left(3.0 \mathrm{~km} \mathrm{~h}^{-1}\right)$. This diminished activity of the muscle pump in the plastered leg is confirmed by our data of the immobilized subjects without filling of the water-pad, showing a markedly reduced venous EF $(30 \%)$ and $\mathrm{EV}[1.3 \pm 0.8 \mathrm{~mL}$ $(100 \mathrm{~mL})^{-1}$ ] compared with healthy controls. Previous studies in healthy subjects, using similar techniques, indicated the venous EF to be $>60 \%$ (van Beekvelt et al. 2000, Nicolaides 2000, Stewart et al. 2004), and EVs to range between 2.0 and $3.0 \mathrm{~mL}(100 \mathrm{~mL})^{-1}$ (Bermudez et al. 1998, Stewart et al. 2004). These data indicate a marked attenuation of muscle pump function during lower leg cast immobilization. An ongoing debate questions, the importance of the skeletal muscle pump function in enhancing flow in skeletal muscles, while it is well-known that when the muscle contracts, the veins within the muscle are compressed and the venous contents are expelled which increases venous return to the heart (Sheriff 2005). It is exactly this compression of the veins that is mimicked by the water-pad in the present study.

A critical question in this study may be whether the water-pad limits lower leg filling. One may argue that increased filling of the water-pad attenuates upright VV and, consequently, affects venous EF. However, data show that maximal VV was not affected by the increasing filling condition of the water-pad.

We hypothesized that the water-pad mobilizes water from the foot to the calf during walking, thereby increasing the pressure around the calf during the standphase and assisting the muscle pump to enhance the venous outflow. The hypothesis was confirmed by the present study as the reduced venous $\mathrm{EV}$ and $\mathrm{EF}$ improved markedly with 41 and $40 \%$, respectively, at $150 \mathrm{~mL}$ filling of the water-pad. This enlargement represents a substantial reversion of the decreased lower leg venous outflow during leg immobilization.

Previously, a pneumatic compression pump has been used to stimulate the venous plexus of the foot and this increased venous flow with $60 \%$ and $189 \%$ in the femoral and popliteal vein, respectively, using duplex- sonography (Bulitta et al. 1996). Electrical stimulation of the calf muscles in an unplastered situation resulted in an EF of $38 \%$ using air plethysmography (Faghri et al. 1997) or $20-40 \%$ increase in venous outflow using echo-Doppler (Kaplan et al. 2002). Klecker \& Theiss (1994) used electrical stimulation of the calf during a below-the-knee plaster. Depending on the strength of the current, an 8.5 -fold increase in maximal flow of the popliteal vein was reported. Although previous methods indicate positive physiological effects, shortcomings of these methods such as applicability in a plaster cast, patients' compliance, as well as short duration of the stimulation techniques hampers the wide application for prevention of deep venous thrombosis. On the contrary, the water-pad investigated in this study overcomes these shortcomings, conveys its effect during walking, and results in a significant and relevant venous emptying during stepping.

Body height had the strongest correlation with the optimum water-pad size, suggesting that the choice for a pad size strongly depends on subject's body height. The results indicate cut-off points at 168 and $182 \mathrm{~cm}$ for increasing water-pad size (small-medium-large). Choosing the incorrect water-pad size or filling condition may lead to discomfort. The choice for a good pad size is important, as data of the non-optimal sized water-pad demonstrate no change in venous EF or EV. However, analysis of the subjects around the cut-off values $( \pm 2 \mathrm{~cm})$ showed an increase in venous ejection and volume using both water-pads, indicating that body heights around these cut-off points are open for subjective interpretation of the optimal size. For the medium sized water-pad ( $n=12)$, an optimal filling of $150 \mathrm{~mL}$ is suggested. Because of small numbers for the small $(n=3)$ and large $(n=6)$ water-pads, optimal filling could only be hypothesized.

\section{Clinical relevance}

Deep venous thrombosis (DVT) is a frequently reported complication (Geerts et al. 1994, Jorgensen et al. 2002, Lassen et al. 2002, Anderson \& Spencer 2003) during lower leg immobilization after fracture, requiring an efficient countermeasure to prevent or decrease its incidence. Previously mentioned drawbacks of lowmolecular-weight-heparin (Kujath et al. 1993, Spannagel \& Kujath 1993, Handoll et al. 2002, Lassen et al. 2002, Schonenberg et al. 2003) or mechanical interventions (Klecker \& Theiss 1994, Bulitta et al. 1996, Kaplan et al. 2002) as a preventive strategy for DVT asks for other methods. Although in healthy subjects the water-pad markedly enhances the venous EV of the lower leg during acute immobilization, future studies should evaluate the effect of the water-pad in cast patients. In these future studies, one should keep in 
mind that the water-pad only works during locomotion or intermittent alterations of pressure under the foot sole. Whether this drawback of the water-pad limits its efficiency is debatable since walking is a frequent daily activity, even for less ambulant subjects such as cast patients.

\section{Limitations}

An important limitation of the present study is that the results of our healthy subjects wearing cast only for a brief period cannot be generalized to cast patients who might actually receive benefit. As we can only hypothesize about the possible effects of the water-pad on the vascular physiology of chronically cast patients, future research is necessary. Nevertheless, in a subpopulation of 10 individuals, one lower leg was immobilized for $24 \mathrm{~h}$ with the use of the water-pad (data not shown). None of the subjects reported any specific discomfort during this period.

In conclusion, the recently developed water-pad in subjects with plaster cast significantly enhances the venous EF and EV of the lower leg during stepping. Counteraction of venous stasis by the water-pad may decrease the incidence of deep venous thrombosis during a prolonged period of immobilization. In addition, the water-pad is an easy applicable method for the patient without demanding additional time or effort to effectively enhance venous EF or volume by imitating the muscle pump.

\section{Conflict of interest}

None of the authors have any conflict of interest.

Somas BV financially supported this study.

\section{References}

Anderson, F.A. Jr \& Spencer, F.A. 2003. Risk factors for venous thromboembolism. Circulation 107 (23 Supp 1), Ig-16.

van Beekvelt, M.C., van Asten, W.N. \& Hopman, M.T. 2000. The effect of electrical stimulation on leg muscle pump activity in spinal cord-injured and able-bodied individuals. Eur J Appl Physiol 82, 510-516.

Bermudez, K., Knudson, M.M., Morabito, D. \& Kessel, O. 1998. Fasciotomy, chronic venous insufficiency, and the calf muscle pump. Arch Surg 133, 1356-1361.

Booth, F.W. 1987. Physiologic and biochemical effects of immobilization on muscle. Clin Orthop 219, 15-20.

Bulitta, C., Kock, H.J., Hanke, J., Sievers, K.W. \& SchmitNeuerburg, K.P. 1996. [Promoting venous return in plaster cast by AV impulse system. A preclinical study]. Unfallchirurgie 22, 145-152.

Faghri, P.D., Van Meerdervort, H.F., Glaser, R.M. \& Figoni, S.F. 1997. Electrical stimulation-induced contraction to reduce blood stasis during arthroplasty. IEEE Trans Rehabil Eng 5, 62-69.

Geerts, W.H., Code, K.I., Jay, R.M., Chen, E. \& Szalai, J.P. 1994. A prospective study of venous thromboembolism after major trauma. N Engl J Med 331, 1601-1606.

Halliwill, J.R., Minson, C.T. \& Joyner, M.J. 2002. Measurement of limb venous compliance in humans: technical considerations and physiological findings. J Appl Physiol 87, 1555-1563.

Handoll, H.H., Farrar, M.J., McBirnie, J., Tytherleigh-Strong, G., Milne, A.A. \& Gillespie, W.J. 2002. Heparin, low molecular weight heparin and physical methods for preventing deep vein thrombosis and pulmonary embolism following surgery for hip fractures. Cochrane Database Syst Rev CD000305.

Hjelmstedt, A. \& Bergvall, U. 1968. Incidence of thrombosis in patients with tibial fractures. Acta Chir Scand 134, 209-218.

Jorgensen, P.S., Warming, T., Hansen, K. et al. 2002. Low molecular weight heparin (Innohep) as thromboprophylaxis in outpatients with a plaster cast: a venografic controlled study. Thromb Res 105, 477-480.

Kaplan, R.E., Czyrny, J.J., Fung, T.S., Unsworth, J.D. \& Hirsh, J. 2002. Electrical foot stimulation and implications for the prevention of venous thromboembolic disease. Thromb Haemost 88, 200-204.

Kitahara, A., Hamaoka, T., Murase, N. et al. 2003. Deterioration of muscle function after 21-day forearm immobilization. Med Sci Sports Exerc 35, 1697-1702.

Klecker, N. \& Theiss, W. 1994. [Transcutaneous electric muscle stimulation - a "new" possibility for the prevention of thrombosis?]. Vasa 23, 23-29

Kock, H.J., Schmit-Neuerburg, K.P., Hanke, J., Rudofsky, G. \& Hirche, H. 1995. Thromboprophylaxis with low-molecular-weight heparin in outpatients with plaster-cast immobilisation of the leg. Lancet 346, 459-461.

Kujath, P., Spannagel, U. \& Habscheid, W. 1993. Incidence and prophylaxis of deep venous thrombosis in outpatients with injury of the lower limb. Haemostasis 23(Suppl. 1), 2026.

Lassen, M.R., Borris, L.C. \& Nakov, R.L. 2002. Use of the low-molecular-weight heparin reviparin to prevent deep-vein thrombosis after leg injury requiring immobilization. $N$ Engl J Med 347, 726-730.

Leppilahti, J. \& Orava, S. 1998. Total Achilles tendon rupture. A review. Sports Med 25, 79-100.

Mammen, E.F. 1992. Pathogenesis of venous thrombosis. Chest 102, 640S-644S.

Micheli, L.J. 1975. Thromboembolic complications of cast immobilization for injuries of the lower extremities. Clin Orthop Relat Res 108, 191-195.

Muntz, J., Scott, D.A., Lloyd, A. \& Egger, M. 2004. Major bleeding rates after prophylaxis against venous thromboembolism: systematic review, meta-analysis, and cost implications. Int J Technol Assess Health Care 20, 405-414.

Nicolaides, A.N. 2000. Investigation of chronic venous insufficiency: a consensus statement (France, March 5-9, 1997). Circulation 102, E126-E163.

Schonenberg, D., van Meeteren, M., Nelissen, R.G., der HorstBruinsma, I.E., Poll, R.G. \& Nurmohamed, M.T. 2003. 
[Thrombosis prevention in orthopaedic surgery: clinical practice in the Netherlands in 2002]. Ned Tijdschr Geneeskd 147, 1856-1860.

Sheriff, D. 2005. Point: the muscle pump raises muscle blood flow during locomotion. I Appl Physiol 99, 371-372 discussion 374-375.

Spannagel, U. \& Kujath, P. 1993. Low molecular weight heparin for the prevention of thromboembolism in outpatients immobilized by plaster cast. Semin Thromb Hemost 19(Suppl. 1), 131-141.

Stewart, J.M., Medow, M.S., Montgomery, L.D. \& McLeod, K. 2004. Decreased skeletal muscle pump activity in patients with postural tachycardia syndrome and low peripheral blood flow. Am J Physiol Heart Circ Physiol 286, H1216H1222.
Sugawara, J., Hayashi, K., Kaneko, F., Yamada, H., Kizuka, T. \& Tanaka, H. 2004. Reductions in basal limb blood flow and lumen diameter after short-term leg casting. Med Sci Sports Exerc 36, 1689-1694.

Warkentin, T.E. \& Kelton, J.G. 1991. Heparin-induced thrombocytopenia. Prog Hemost Thromb 10, 1-34.

Warkentin, T.E., Levine, M.N., Hirsh, J. et al. 1995. Heparininduced thrombocytopenia in patients treated with lowmolecular-weight heparin or unfractionated heparin. N Engl J Med 332, 1330-1335.

Zagrodnick, J. \& Kaufner, H.K. 1990. [Ambulatory thromboembolism prevention in traumatology using self-injection of heparin]. Unfallchirurg 93, 331-333. 